\title{
A universal density slope - velocity anisotropy relation for relaxed structures
}

\author{
Steen H. Hansen, Ben Moore \\ University of Zurich, Winterthurerstrasse 190, 8057 Zurich, Switzerland
}

\begin{abstract}
We identify a universal relation between the radial density slope $\alpha(r)$ and the velocity anisotropy $\beta(r)$ for equilibrated structures. This relation holds for a variety of systems, including disk galaxy mergers, spherical collapses, cold dark matter (CDM) halos both with and without cooling. We argue that the shape of the relation is reasonable from fundamental principles when the dark matter or stars are assumed to obey Tsallis statistics, and in that case we fit the $\alpha-\beta$ relation with just one free parameter. One can use this result to close the Jeans equations, for example to construct mass models of elliptical galaxies from observational data or to tune dark matter direct detection experiments. We also predict the asymptotic central slope and anisotropy of CDM halos to be approximately -1 and 0 .
\end{abstract}

Key words:

\section{Introduction}

Gravitating equilibrium structures must satisfy the Jeans equations, however these are generally difficult to solve - especially for multi-component or flattened systems. One of the main difficulties is that there is no clear connection between the density and the components of the velocity dispersion tensor. Even for the simplest gravitationally bound systems of collisionless particles one must make strong assumptions to find a solution. One example hereof is the central density profile of dark matter structures, which has still not been derived directly from the collisionless Boltzmann equation. Instead one must resort to N-body simulations which have been used to demonstrate a universality amongst the density profiles of CDM structures, see e.g. (Diemand, Moore \& Stadel 2004), and references within. Only when making strong assumptions about a connection between the density and the velocity dispersion can one find analytical solutions (Binney \& Tremaine 1987). E.g. under the assumption that the phase-space density is a power-law in radius can 


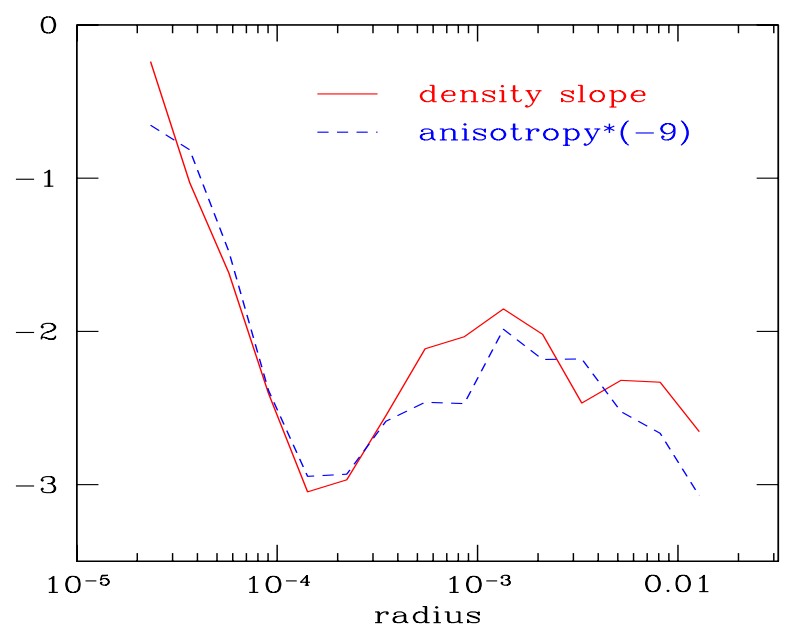

Fig. 1. The radial anisotropy and density profile slope of an equilibrated dark matter structure where $5 \%$ of the particles are allowed to cool in order to mimic baryons. The red (full) line is the density slope of all gravitating matter, and the blue (dashed) line is a simple linear function of the DM anisotropy.

one solve for the density profile (Hansen 2004, Austin et al. 2005, Dehnen \& McLaughlin 2005). Furthermore, if one assumes a given form for the density profile and assumes a given radial dependence of the anisotropy, then one can derive the radial behaviour of mass, potential and velocity dispersion, see e.g. (Lokas \& Mamon 2002) for references. Similarly, it has also been suggested that the velocity anisotropy should follow a universal profile in radius for dark matter structures (Cole \& Lacey 1996, Carlberg et al. 1997), however these relations are not generally valid, as will be shown in Fig. 1.

We will here address a related question, namely whether there is a connection between the radial derivative of the density profile, the density slope $\alpha(r)$,

$$
\alpha(r) \equiv \frac{d \ln \rho}{d \ln r}
$$

and the velocity anisotropy $\beta(r)$

$$
\beta(r) \equiv 1-\frac{\sigma_{t}^{2}}{\sigma_{r}^{2}},
$$

where $\rho(r)$ is the density, and $\sigma_{t}^{2}$ and $\sigma_{r}^{2}$ are the one dimensional tangential and radial velocity dispersions. We will show that this connection is universal (within the scatter from the numerical simulations), and we will argue that such a connection is reasonable from a fundamental statistical mechanics point of view. We find that the $\alpha-\beta$ relation can be argued (or maybe eventually derived) from first principles, with only one free parameter. 


\section{$2 \quad$ A relation between $\alpha$ and $\beta$}

In order to address the question of a possible relation between the density slope $\alpha$ and the velocity anisotropy $\beta$ we first consider a result from an Nbody simulation. This simulation follows the hierarchical formation of a dark matter structure where $5 \%$ of the particles are allowed to cool, mimicking the behaviour of baryons. The reason for considering this specific simulation is that it is remarkably complex and captures many of the physical processes that may arise during the formation of galactic systems. The final dark matter structure is built up of many hundreds of minor and major mergers of small halos. The resulting triaxial structure is modified by the effects of dissipating particles, deepening the potential, altering the orbital classes of dark matter particles and changing the global shape of the system. The final system does not follow the universal density profile and has a complex distribution of dark matter and particles allowed to cool - the latter dominate the potential within a few percent of the virial radius.

The question of resolved region and whether the structure at a given (large) radius is equilibrated will be discussed later. The result is presented in Fig. 1, where the red (full) line is the density slope of all the matter present. On the same figure we present a simple linear function of the anisotropy, $-9 \cdot \beta$, as a blue (dashed) line. This demonstrates a clear connection between the density slope and the anisotropy. If a relation reveals itself in this highly complex simulation, then we expect that a similar relation might hold for any relaxed and equilibrated structure.

It is important to clarify that whereas universality has previously been suggested both for the radial density profile (Navarro, Frenk \& White 1996, Moore et al. 1998) and the radial anisotropy profile (Cole \& Lacey 1996, Carlberg et al. 1997), then the simulated density and anisotropy profiles presented in Fig. 1 clearly do not follow such universal behaviour: this non-universality at intermediate radii is seen as a dip in Fig. 1, which is absent in the previously suggested universal profiles. Nevertheless, the anisotropy and density slope have a clear correlation for all radii.

When we discuss relaxed structures, we mean that the system has undergone rapid processes of violent restructuring to attain a new quasi-equilibrium state, such as a merger, i.e. an elliptical galaxy resulting from the merger between two disk galaxies. A true equilibrium never occurs for any astrophysical system since they are not ideal fluids, rather they are made of massive objects such as stars (or dark matter particles) which undergo continuous short and long range interactions. Over very long timescales this collisionality may cause the central regions to collapse and the outer particles to disperse. For a spiral galaxy, the stars will evolve from a disk into a spheroidal structure over a 
timescale of $\sim 10-100$ Hubble times.

\section{A universal $\alpha-\beta$ relation}

Let us now explore the properties of four additional different simulations.

(i) Firstly we consider a spherical collapse calculation. We place one million particles in a spherical distribution with an initial $r^{-1}$ density profile. The system has unit mass an initial unit size and the particles have zero velocity. We run the simulation for over 10 dynamical times $t_{d y n} \propto(G \rho)^{-0.5}$. The system maintains its symmetry only until the first shells cross in the central region. The radial orbit instability occurs and the infalling shells of particles undergo strong scattering from a central bar-like structure. The final equilibrium structure has a half mass radius of about 0.1 and is prolate with axial ratios of about $2: 1$. The density profile has a slope of -1.3 in the innermost resolved region - similar to that found within the cosmological simulations of CDM halos.

(ii) Our second study starts from two identical equilibrium NFW structures each with zero anisotropy. These are constructed using the technique described in (Kazantzidis, Magorrian \& Moore 2004). Each halo has one million particles and has a mass of $10^{12} M_{\odot}$ and a concentration parameter $c_{\mathrm{nfw}}=10$. We carry out two simulations in which the halos collide head on producing a prolate structure, and a merger with angular momentum which produces an oblate structure. The simulations are run for 10 Gyrs to allow the merger remnant to attain equilibrium, see (Moore et al. 2004) for further details.

(iii) We carry out a merger between two disk galaxies that are constructed to be similar to a late type disk galaxy such as M32. The halo is initially NFW but adiabatically contracted due to the presence of an exponential disk of stars. The two disks are placed on a parabolic inclined orbit typical of those found within cosmological simulations. We analyse the simulation after 5 Gyrs after which the system resembles a relaxed elliptical galaxy.

(iv) Finally we take a high resolution cosmological simulation of a cold dark matter halo which is resolved with over ten million particles. This is one of the cluster mass halos from the study of (Diemand, Moore \& Stadel 2004). The density profile attains a slope of -1.3 in the innermost resolved region.

For each of these simulations, which have been allowed to approximately equilibrate, we extract the density profile and the radial dependence of the anisotropy. The results are shown in Fig. 2 as symbols. Surprisingly, there seems to be a clear trend, namely that for these completely different simu- 


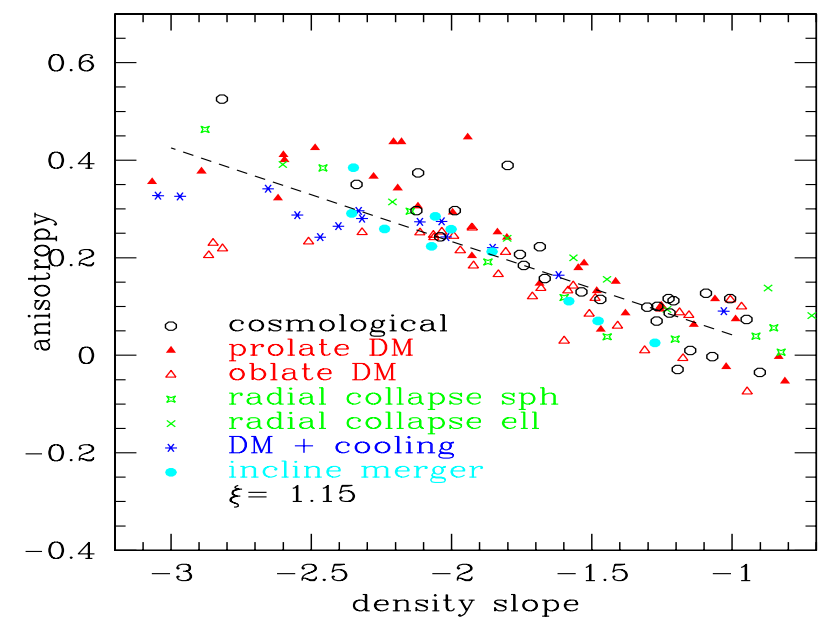

Fig. 2. The final density slope versus velocity anisotropy for the six different simulations (coloured symbols) discussed in the text. For the spherical collapse calculation we plot the result using both spherical and elliptical bins. A general relation is clear with the slope in the range -3 to -1 . The shape of the relation is reasonably well fit with 2 parameters, $\beta=\eta_{1}-\eta_{2} \alpha$, where approximately $-0.45<\eta_{1}<0.05$ and $0.1<\eta_{2}<0.35$. The dashed (black) line is a one parameter fit as discussed in section 4 , with the parameter $\xi=1.15$.

lations the relation between the slope of the mass profile and the anisotropy parameter of the particles is the same. The simulations are roughly resolved to the radius where the slope is approximately -1 , and should not be trusted for more shallow profiles. This resolution limit is set by the number of particles in the central regions - we do not know if the correlation we find is affected by the numerical relaxation processes that occur where particle-particle encounters are important. For slopes steeper than $\approx-2.5$ the particles have often not had time to equilibrate fully.

In principle, the anisotropy parameter could have any value in the range $-\infty<$ $\beta \leq 1$. Isotropic orbits have $\beta=0$ and positive values correspond to more radial orbits. On Fig. 2 we see that the relations is approximately fit with $\beta=\eta_{1}-\eta_{2} \alpha$. More detailed simulations and convergence tests are in progress to clarify the best fitting relation and to investigate the cause of the scatter. It will be interesting to explore how much "violent relaxation" is required to force an existing equilibrium structure to lie on this correlation, and what the driving force behind establishing the correlation is. The time evolution of the radial infall simulation allows us to compare the change of anisotropy with the change in the mass profile. Even though naively, one might have expected that the density profile should first manifest itself and then only later the anisotropy should appear, it seems that the two come hand in hand. As soon as a given density profile exists (and is approximately equilibrated) then the anisotropy exists as well, and sits near the $\alpha-\beta$ relation. We also point out that the orbital structure of the models does not play an important role in 
changing the observed correlation. The halo merger simulations produced a prolate halo with over 90 percent of the particles on box orbits whilst the oblate halo was primarily tube and loop orbits, yet both models lie on the $\alpha-\beta$ relation.

\section{Discussion}

The relation identified in the previous sections turns out to be rather nontrivial to explain, and the intuitive arguments of this section will make it evident that there is still much work to be done.

For a classical gas the velocity dispersion is like a temperature. If you have a box of classical particles then the velocity dispersion seen from one side of the box will be the same as seen from another side of the box. For the dark matter structures the anisotropy is therefore like having different temperatures when considering the radial and tangential directions. The question we are posing is, why is this related to the density profile? Since these completely different simulations described above seem to give the same relation, then the reason must be of fundamental nature. Let us therefore consider the velocity distribution function, $f(v)$.

The existence of a non-zero anisotropy implies that the velocity distribution function (VDF) in the radial direction, $f_{r}$, differs from the VDF in the tangential direction, $f_{t}$. Intuitively one may in fact expect $f_{r}$ and $f_{t}$ to differ, simply because the structure is in equilibrium which implies that the VDF's are constant in shape and magnitude. That is seen as follows: particles moving tangentially experience no change in energy, so the conservation of $f_{t}$ is trivial and may be obtained by virtually any shape of $f_{t}$. The height of $f_{t}$ is fixed by the density which is constant along the tangential direction. Contrariwise, particles moving radially will both be changing kinetic energy as well as moving to a region with a different density, thus both the shape and magnitude of $f_{r}$ is non-trivial to conserve. This puts strong constraints on the shape of the radial VDF. We thus see that there are strong constraints on the shape of $f_{r}$, and virtually no constraints on $f_{t}$. There is therefore very little reason to expect isotropy. Now, let us quantify these statements.

It is well known that for a given radial density profile (and zero anisotropy) one can use the Eddington inversion method to extract the velocity distribution function, $f(v)$, at different radial bins. The shape of this distribution function can be obtained analytically for sufficiently simple structures, and in particular for isotropic structures with constant density slope the shape is the Tsallis distribution function, $f(v, q)$, which depends on the non-extensive index $q$ (Tsallis 1988, Plastino \& Plastino 1993). In fact, a simple connec- 
tion between this non-extensive parameter, $q$, and the density slope has been derived by (Hansen et al. 2005)

$$
\alpha=\frac{6 q-10}{3-q}
$$

where typically $0<q<5 / 3$. This equation strictly speaking only holds for isotropic structures. However, the Eddington inversion used to derive this equation is only referring to the radial component of the velocity distribution and simply assumes that the tangential component is identical to the radial component. We will now use eq. (3) to argue that there really should be a difference between the radial and tangential distribution functions, and that real structures therefore should be anisotropic. To test the accuracy of our arguments below, one should derive a connection similar to eq. (3) for anisotropic structures, and if that connection would be similar to eq. (3), then our arguments are reliable.

From the expression in eq. (3) it is clear that for constant density $(\alpha=0)$ one has $q=5 / 3$. Thus, the tangential velocity distribution function will have a shape given by $q=5 / 3$, whereas the radial velocity distribution function will generally have a shape given by a different $q$. We therefore understand that the particle distributions generally are different in the radial and the tangential directions. It is reasonable to speculate that it really is the changing potential energy being the driving force between the difference in the radial and tangential direction.

Recently it has indeed been confirmed that the velocity distribution function for the radial and tangential part indeed can be described by using different $q$ 's as suggested above (Hansen et al. 2005). In particular was it shown that the bulk of the tangential distribution function indeed should be described by $q=5 / 3$ as we have just argued. This is strong support that our arguments presented above are fairly reliable.

One can directly derive the fraction between velocity dispersions for different $q$ 's. When the energy of the particles is trivially given as $E \sim v^{2}$, that is, if the potential energy was constant, then the result is

$$
\frac{\sigma_{t}^{2}}{\sigma_{r}^{2}(q)}=\frac{S(t)}{S(r)} \frac{A(t)}{\zeta(t)} \frac{\zeta(r)}{A(r)},
$$

with

$$
S(q, D)=\frac{2 D}{(D+2)-D q}
$$


where $\zeta$ is the Lagrange multiplier and should cancel, $A=1+(1-q) D / 2$ (Boghosian 1999), and we have explicitely written the $r$ and $t$ for radial and tangential. These equations only hold when the distributions, radial and tangential separately, can be approximated by the Tsallis shape. The S-term was previously derived for 3 dimensions (Silva \& Alcaniz 2003), and the q-dependence of A may be important for cluster temperature determination (Hansen 2005). Using eq. (3) we have

$$
A(\alpha, D)=\frac{\alpha(1-D)+(6-2 D)}{6+\alpha} .
$$

Since we are considering 1-dimensional velocity dispersions we have $D=1$ and one finds

$$
\frac{A(t)}{A(r)}=\frac{6+\alpha}{6} .
$$

In the simplest of all worlds one would indeed have $E \sim v^{2}$, in which case the S-term in eq. (5) would exactly cancel with eq. (7), and we would have identical zero anisotropy everywhere. One can, however, imagine more complicated situations where this does not happen since the potential energy changes as function of radius, so that the S-term would look different. Phenomenologically we parameterise our ignorance with an unknown constant, $\xi$, and we therefore conclude that the anisotropy may look like

$$
\beta(\alpha)=1-\xi(1+\alpha / 6)
$$

The points on Fig. 2 are approximated with $\xi \approx 1.15$, as shown with the dashed (black) line.

The first obvious objection is that whereas a connection between the slope and the anisotropy is clear from Fig. 1, there is a large scatter in Fig. 2. It may in fact be that the relation is not universal after all, in which case it would make no sense trying to derive it from first principles. To this end one must keep in mind the large range that our simulations span. They include both cosmological simulations and also controlled colliding initially isotropic models $(\beta=0)$ and radial collapse with $\beta=1$. It would be an impressive coincidence if these simulations would conspire to accidentally land near the same line in $\alpha-\beta$ space. At a given value of the density profile the scatter in the anisotropy parameter is about 0.2 .

The second objection is that it is possible to create a structure e.g. with a Hernquist density profile and with zero anisotropy everywhere, which is stable, so the suggested $\alpha-\beta$ relation cannot be a necessary criteria for all 
equilibrium systems. This is similar to the statement that it is possible to arrange a configuration of classical particles in a box which all move exactly in the $x$-direction only. This configuration is in principle also stable, however, any small perturbation (e.g. inclusion of gravity) will perturb the system, and soon the classical particles will achieve an isotropic distribution. Similarly, the suggested Hernquist structure may (and we suggest ${ }^{1}$ that it will) move away from the initial zero anisotropy, and flow towards the line in $\alpha-\beta$ space, when the system is exposed to significant perturbations, such as merging of substructures. A large perturbation of the artificially constructed classical box of particles will rapidly lead to equilibration, whereas a small perturbation will only slowly lead to equilibration. Similarly, we expect that a larger perturbation will drive any equilibrium structure towards the universal $\alpha-\beta$ line.

The third objection is our argued fitting formula, eq. (8). This must clearly break down for small density slopes. The reason is that when the slope is zero, then there is basically no difference (from the point of view of the velocity distribution function as explained above) of going in the radial or the tangential direction, and hence the anisotropy may go to zero. Furthermore, eq. (3) was derived for isotropic structures. It may, however, be that the correct relation between $\alpha$ and $q$ should depend on the anisotropy as well. In that case the arguments above will change.

We note that our finding has consequences for dark matter detection, since the Earth is expected to be near $\alpha \approx-1.3$ which implies $\beta \approx 0.1$. First the existence of an anisotropy will alter the annual modulation (Vergados 1999, Evans, Carollo \& de Zeeuw 2000), and second the anisotropy will also allow for a 'non-annual-modulation' differentiation between signals tangentially and radially w.r.t. the galaxy center with a directional sensitive DM detector.

We can finally combine our findings with known results, in order to predict the central slope and anisotropy of equilibrated dark matter structures. One can find a very general bound on the central anisotropy as function of the central density slope, $\beta \leq \gamma / 2$, which is valid for all systems obeying the Jeans equation (An \& Evans 2005). Much stronger bounds naturally appear when considering more restrictive systems. When assuming that phase-space density is a pure power-law in radius (as observed in systems from cosmological simulations (Taylor \& Navarro 2001)), then the bound on the central slope as a function of the anisotropy is instead (Hansen 2004)

$$
\alpha(0) \leq-(1+\beta(0))
$$

$\overline{1}$ We performed a controlled experiment, where each individual particles in an initially stable Herquist structure is perturbed slightly and then the system is allowed to equilibrate, repeatedly. These perturbations thus act like a non-zero term on the r.h.s. of the boltzmann equation, and hence antonov's laws of stability may not apply. We indeed observe that the structure slowly moves towards the $\alpha-\beta$ line. 
which together with the $\alpha-\beta$ relation implies that the central values are given by $(\alpha(0), \beta(0)) \approx(-1,0)$. One should keep in mind the scatter in Fig. 2 , which implies that this bound still has significant uncertainties ${ }^{2}$.

\section{Conclusions}

We have identified a relation between the density slope and the velocity dispersion for dark matter structures, and we suggest that this relation may be universal. We support this with a set of completely different simulations, which all land near this $\alpha-\beta$ relation. We argue that the relation is reasonable from a fundamental statistical mechanics point of view, when the star or dark matter distribution functions are to be described by Tsallis statistics, and the relation is in that case fit with just one free parameter. We use our result to predict that the central slope and velocity anisotropy are approximately $(\alpha(0), \beta(0)) \approx(-1,0)$.

This remarkable correlation implies that in all systems that have undergone a violent relaxation process, the anisotropies of all components (e.g. stars and dark matter) are identical to each other. Utilising this fact allows a unique solution to the Jeans equation to be established for any given system. No longer is the anisotropy a free parameter - it can be related to the local slope of the total density profile. This will allow unique mass models to be constructed for elliptical galaxies for example. Given good enough data, one could measure the anisotropy of the stars in such a system to infer the structure of the dark matter halo. It can also be used to tune direct detection experiments.

\section{Acknowledgements}

It is a pleasure to thank Juerg Diemand and Joachim Stadel for discussions and simulations. We thank Wyn Evans for insightful comments. SHH thanks the Tomalla foundation for financial support.

$\overline{2}$ Recently Dehnen \& McLaughlin (2005) refined the analysis of Hansen (2004), and they find the central slope to be connected to the central anisotropy as $\gamma_{0}=$ $-\left(7+10 \beta_{0}\right) / 9$, also under the assumption that phase-space density is a power-law in radius as observed in N-body simulations. When using this analytical result together with the numerical findings of this paper do we conclude that the central anisotropy is close to zero, and the central density slope is close to -0.8 . 


\section{References}

An, J. H. \& Evans, N. W. 2005, submitted to ApJ

Austin, C. G. et al 2005, arXiv:astro-ph/0506571

Binney, J. \& Tremaine, S. 1987, Galactic Dynamics, Princeton University Press

Boghosian, B. M. 1999, Braz. J. Phys., 29, 91

Carlberg, R. G. 1997, ApJ, 485, L13

Cole, S. \& Lacey, C. 1996, MRNAS, 281, 716

Dehnen, W. \& McLaughlin, D. E. 2005, arXiv:astro-ph/0506528.

Diemand, J., Moore, B. \& Stadel, J. 2004, MNRAS, 353, 624

Evans, N. W., Carollo, C. M. \& de Zeeuw, P. T. 2000, MNRAS, 318, 1131

Hansen, S. H. 2004, MNRAS, 352, L41

Hansen, S. H. 2005, New Astron, 10, 371

Hansen, S. H., Egli, D., Hollenstein, L. \& Salzmann, C. 2005, New Astron, 10, 379

Hansen, S. H., Moore, B., Zemp, M. \& Stadel, J 2005, arXiv:astro-ph/0505420.

Kazantzidis, S., Magorrian, J. \& Moore, B. 2004, ApJ, 601, 37

Lokas, E. L. \& Mamon, G. A. 2001, MNRAS, 321, 155

Moore, B., Governato, F., Quinn, T., Stadel, J. \& Lake, G. 1998, ApJ, 499, 5

Moore, B., Kazantzidis, S., Diemand, J. \& Stadel, J. 2004, MNRAS, 354,522

Navarro, J. F., Frenk, C. S. \& White, S. D. M. 1996, ApJ, 462, 563

Plastino, A. R. \& Plastino, A. 1993, PLA, 173, 384

Silva, R. \& Alcaniz, J. S. 2003, PLA, 313, 393

Taylor, J. E. \& Navarro, J. F. 2001, ApJ, 563483

Tsallis, C. 1988, J. Stat. Phys., 52, 479

Vergados, J. D. 1999, PRL, 83, 3597 